PROCEEDINGS OF THE

AMERICAN MATHEMATICAL SOCIETY

Volume 134, Number 8 , Pages 2251-2258

S 0002-9939(06)08587-X

Article electronically published on March 14, 2006

\title{
CONVERGENCE OF A SINGULAR EULER-POISSON APPROXIMATION OF THE INCOMPRESSIBLE NAVIER-STOKES EQUATIONS
}

\author{
R. NATALINI AND F. ROUSSET \\ (Communicated by Suncica Canic)
}

\begin{abstract}
In this note, we rigorously justify a singular approximation of the incompressible Navier-Stokes equations. Our approximation combines two classical approximations of the incompressible Euler equations: a standard relaxation approximation, but with a diffusive scaling, and the Euler-Poisson equations in the quasineutral regime.
\end{abstract}

\section{INTRODUCTION}

We shall consider the following system:

$$
\left\{\begin{array}{l}
\partial_{t} u^{\varepsilon}+\operatorname{div} V^{\varepsilon}=\nabla \varphi^{\varepsilon}, \\
\varepsilon \partial_{t} V^{\varepsilon}+a^{2} \nabla u^{\varepsilon}=u^{\varepsilon} \otimes u^{\varepsilon}-V^{\varepsilon}, \\
\partial_{t} \rho^{\varepsilon}+\operatorname{div}\left(\rho^{\varepsilon} u^{\varepsilon}\right)=0, \\
\Delta \varphi^{\varepsilon}=\frac{\rho^{\varepsilon}-1}{\varepsilon},
\end{array}\right.
$$

for $t>0, x \in \mathbb{T}^{d}, u^{\varepsilon} \in \mathbb{R}^{d}, V^{\varepsilon} \in \mathbb{R}^{d, d}, \rho^{\varepsilon} \in \mathbb{R}$. Here $\varepsilon>0$ is a small parameter and $a$ is a non zero constant value. To uniquely solve the Poisson equation, we add the condition $\int_{\mathbb{T}^{d}} \rho=1$. Passing to the limit when $\varepsilon$ goes to zero, it is easy to see, at least at a very formal level, that $\left(\rho^{\varepsilon}, u^{\varepsilon}, V^{\varepsilon}\right)$ tends to $\left(\rho^{N S}, u^{N S}, V^{N S}\right)$, where

$$
\rho^{N S}=1, V^{N S}=u^{N S} \otimes u^{N S}-a^{2} \nabla u^{N S}
$$

and

$$
\left\{\begin{array}{l}
\partial_{t} u^{N S}+\operatorname{div}\left(u^{N S} \otimes u^{N S}\right)=a^{2} \Delta u^{N S}+\nabla \varphi^{N S} \\
\operatorname{div} u^{N S}=0 .
\end{array}\right.
$$

Received by the editors February 1, 2005.

2000 Mathematics Subject Classification. Primary 35Q30; Secondary 76D05.

Key words and phrases. Incompressible Navier-Stokes equations, quasineutral regime, EulerPoisson equations, diffusive relaxation approximations, hyperbolic singular perturbations.

The research activity reported in this paper has been partially conducted within the European Union RTN HYKE project: HPRN-CT-2002-00282.

(C)2006 American Mathematical Society Reverts to public domain 28 years from publication 
In other words, $u^{N S}$ is a solution of the incompressible Navier-Stokes equations. The aim of this note is to give a rigorous justification to this formal computation. We shall prove the following result.

Theorem 1. Let $u^{N S}$ be a solution of the Navier-Stokes equations (3) such that $u^{N S} \in C\left([0, T], H^{m+3}\left(\mathbb{T}^{d}\right)\right)$ and $\int_{\mathbb{T}^{d}} u^{N S}=0$ for $d \leq 3$ and $m>1+d / 2$. Assume that the initial value $\left(u_{0}^{\varepsilon}, V_{0}^{\varepsilon}, \rho_{0}^{\varepsilon}\right) \in H^{m+1}$ of (1) is such that $\int_{\mathbb{T}^{d}} \rho_{0}^{\varepsilon}=1, \int_{\mathbb{T}^{d}} u_{0}^{\varepsilon}=0$ and

$$
\alpha_{m}(\varepsilon):=\left\|u_{0}^{\varepsilon}-u_{/ t=0}^{N S}\right\|_{H^{m+1}}^{2}+\frac{1}{\varepsilon}\left\|\rho_{0}^{\varepsilon}-1\right\|_{H^{m}}^{2}+\varepsilon\left\|V_{0}^{\varepsilon}-V_{/ t=0}^{N S}\right\|_{H^{m+1}}^{2} \rightarrow 0
$$

when $\varepsilon$ goes to zero. Then there exist $\varepsilon_{0}$ and $C_{T}$ such that for $0<\varepsilon \leq \varepsilon_{0}$ there is a strong solution $\left(\rho^{\varepsilon}, u^{\varepsilon}, V^{\varepsilon}\right) \in C\left([0, T], H^{m+1}\right)$ of (1) such that

$$
\left\|u^{\varepsilon}(t)-u^{N S}(t)\right\|_{H^{m+1}}^{2}+\frac{1}{\varepsilon}\left\|\rho^{\varepsilon}(t)-1\right\|_{H^{m}}^{2} \leq C_{T}\left(\varepsilon^{2}+\alpha_{m}(\varepsilon)\right), \forall t \in[0, T] .
$$

System (1) combines the features of the diffusive relaxation approximation of parabolic systems and of the quasineutral limit of the Euler-Poisson equation towards the incompressible Euler equation. These two problems were extensively studied by various methods.

Let us recall that the diffusive scaling $\left(\frac{x}{\sqrt{\varepsilon}}, \frac{t}{\varepsilon}\right)$ has been largely investigated in the framework of hydrodynamic limits of the Boltzmann equations [4] and in the analysis of hyperbolic-parabolic relaxation limits for weak solutions of hyperbolic systems of balance laws with strongly diffusive source terms by means of compensated compactness techniques by Marcati and collaborators [15, 16, 9]. Under the same scaling, general (possibly degenerate) parabolic equations in multi-D have been approximated by semilinear hyperbolic equations; see [5, 2].

Concerning the quasineutral limit, which consists in the limit of vanishing viscosity for the Poisson part of the system, there are results for various specific models. In particular, this limit has been performed for the Vlasov-Poisson system by Brenier [6, Grenier [11, 12, and in the one-dimensional and isothermal Euler-Poisson system by Cordier and Grenier [8]. We refer to [14, [18] and references therein for more recent contributions.

The main focus in the present note is on the use of hyperbolic energy methods for studying incompressible fluids. In this regard, our system can also be seen as a refinement of the relaxation system studied in [7, whose techniques however were restricted to the two-dimensional case. In particular our result gives a rate of convergence in strong $H^{s}$ norm of the solution of the singular system towards a strong solution of the incompressible Navier-Stokes equation. Hyperbolic ideas have also been used in the weak solutions framework by Donatelli and Marcati to deal with the diffusive relaxation of hyperbolic approximations towards Leray solutions of the incompressible Navier-Stokes equation 10. Finally, similar approximations have been considered as reduced kinetic models in [3] and on a rigorous basis in [13], to design effective numerical schemes for incompressible fluids.

\section{Proof of Theorem 1}

First, let us set

$$
u=u^{\varepsilon}-u^{N S}, V=V^{\varepsilon}-V^{N S}, \varphi=\varphi^{\varepsilon}-\varphi^{N S}, \rho=\rho^{\varepsilon}-1 .
$$


Since the pressure $\varphi^{N S}$ in the incompressible Navier-Stokes equation is given by

$$
\Delta \varphi^{N S}=\nabla\left(u^{N S} \cdot \nabla u^{N S}\right)=\nabla u^{N S} \cdot \nabla u^{N S},
$$

the vector $(\rho, u, V, \varphi)$ solves the system

$$
\left\{\begin{array}{l}
\partial_{t} u+\operatorname{div} V=\nabla \varphi, \\
\varepsilon \partial_{t} V+a^{2} \nabla u=u^{N S} \otimes u+u \otimes u^{N S}-V+u \otimes u+\varepsilon \partial_{t} V^{N S}, \\
\partial_{t} \rho+\operatorname{div} u+u^{N S} \cdot \nabla \rho+\operatorname{div}(\rho u)=0, \\
\Delta \varphi=\frac{\rho}{\varepsilon}-\nabla u^{N S} \cdot \nabla u^{N S} .
\end{array}\right.
$$

As in [14, we make the following change of unknowns:

$$
\begin{gathered}
d=\operatorname{div} u, \omega=\operatorname{curl} u \\
D_{i}=(\operatorname{div} V)_{i}=\sum_{j} \partial_{j} V_{i j}, \Omega_{i j}=(\operatorname{curl} V)_{i j}=\partial_{i} V_{j}-\partial_{j} V_{i} .
\end{gathered}
$$

By using the last equation in (5D) and the fact that $\Delta u=\nabla d-\operatorname{curl} \omega$, we get the following system:

$$
\left\{\begin{aligned}
\partial_{t} d+\operatorname{div} D= & \frac{\rho}{\varepsilon}-\nabla u^{N S} \cdot \nabla u^{N S}, \\
\partial_{t} \omega+\operatorname{curl} D= & 0 \\
\varepsilon \partial_{t} D+a^{2}(\nabla d-\operatorname{curl} \omega)= & -D+\operatorname{div}\left(u^{N S} \otimes u+u \otimes u^{N S}\right) \\
& -\operatorname{div}(u \otimes u)+\varepsilon \partial_{t}\left(\operatorname{div} V^{N S}\right), \\
\varepsilon \partial_{t} \Omega= & -\Omega+\operatorname{curl}\left(u^{N S} \otimes u+u \otimes u^{N S}\right) \\
& +\varepsilon \partial_{t} \operatorname{curl} V^{N S}-\operatorname{curl}(u \otimes u), \\
\partial_{t} \rho+u^{N S} \cdot \nabla \rho= & -d-\operatorname{div}(\rho u) .
\end{aligned}\right.
$$

This last system can be written as a singular perturbation of a quasilinear symmetrizable hyperbolic system. Setting $\mathcal{U}=(d, \omega, D, \Omega, \rho)$ yields

$$
\mathcal{A}_{0}^{\varepsilon} \partial_{t} \mathcal{U}+\mathcal{A}\left(t, x, \partial_{x}\right) \mathcal{U}+\frac{1}{\varepsilon} \mathcal{K}^{\varepsilon} \mathcal{U}+\mathcal{D} \mathcal{U}+\mathcal{N}\left(\mathcal{U}, \partial_{x} \mathcal{U}\right)=\mathcal{L}\left(t, x, \partial_{x}\right) \mathcal{U}+\varepsilon R
$$


where

$$
\begin{aligned}
& \mathcal{A}_{0}^{\varepsilon}=\operatorname{diag}(1,1, \varepsilon, \varepsilon, 1), \mathcal{D}=\operatorname{diag}(0,0,1,1,0), \\
& \mathcal{A}\left(t, x, \partial_{x}\right)=\left(\begin{array}{ccccc}
0 & 0 & \operatorname{div} & 0 & 0 \\
0 & 0 & \operatorname{curl} & 0 & 0 \\
a^{2} \nabla & -a^{2} \text { curl } & 0 & 0 & 0 \\
0 & 0 & 0 & 0 & 0 \\
0 & 0 & 0 & 0 & u^{N S} \cdot \nabla
\end{array}\right), \\
& \mathcal{K}^{\varepsilon}=\left(\begin{array}{ccccc}
0 & 0 & 0 & 0 & -1 \\
0 & 0 & 0 & 0 & 0 \\
0 & 0 & 0 & 0 & 0 \\
0 & 0 & 0 & 0 & 0 \\
\varepsilon & 0 & 0 & 0 & 0
\end{array}\right) \\
& \mathcal{L}\left(t, x, \partial_{x}\right) \mathcal{U}=\left(\begin{array}{l}
0 \\
0 \\
\operatorname{div}\left(u^{N S} \otimes u+u \otimes u^{N S}\right) \\
\operatorname{curl}\left(u^{N S} \otimes u+u \otimes u^{N S}\right) \\
0
\end{array}\right), \\
& \mathcal{N}\left(\mathcal{U}, \partial_{x} \mathcal{U}\right)=\left(\begin{array}{c}
0 \\
0 \\
\operatorname{div}(u \otimes u) \\
\operatorname{curl}(u \otimes u) \\
\operatorname{div}(\rho u)
\end{array}\right), R=\left(\begin{array}{l}
-\nabla u^{N S} \cdot \nabla u^{N S} \\
0 \\
\partial_{t} \operatorname{div}\left(V^{N S}\right) \\
\partial_{t} \operatorname{curl}\left(V^{N S}\right) \\
0
\end{array}\right) .
\end{aligned}
$$

Let us remark that $\mathcal{L}$ is a zero order operator when it acts on $\mathcal{U}$. The nonlinear term $\mathcal{N}$ can be split into

$$
\mathcal{N}\left(\mathcal{U}, \partial_{x} \mathcal{U}\right)=\mathcal{S}(\mathcal{U})+\mathcal{Q}\left(\mathcal{U}, \partial_{x}\right) \mathcal{U}
$$

where

$$
\mathcal{S}(\mathcal{U})=\left(\begin{array}{l}
0 \\
0 \\
\operatorname{div}(u \otimes u) \\
\operatorname{curl}(u \otimes u) \\
\rho \operatorname{div} u
\end{array}\right)
$$

is a semilinear term (still considered as acting on $\mathcal{U})$ and $\mathcal{Q}\left(\mathcal{U}, \partial_{x}\right) \mathcal{U}$ is a quasilinear term with

$$
\mathcal{Q}\left(\mathcal{U}, \partial_{x}\right) \mathcal{U}=\left(\begin{array}{l}
0 \\
0 \\
0 \\
0 \\
u \cdot \nabla \rho
\end{array}\right)
$$

Let us set

$$
\Sigma^{\varepsilon}=\operatorname{diag}\left(a^{2}, a^{2} I_{d}, I_{d}, I_{d \times d}, a^{2} / \varepsilon\right),
$$


and for $|\alpha| \leq m$

(7)

$$
\begin{aligned}
E_{\alpha, m}^{\varepsilon}(t)= & \frac{1}{2}\left(\Sigma^{\varepsilon} \mathcal{A}_{0}^{\varepsilon} \partial^{\alpha} \mathcal{U}, \partial^{\alpha} \mathcal{U}\right) \\
= & \frac{1}{2}\left(a^{2}\left\|\partial^{\alpha} d\right\|^{2}+a^{2}\left\|\partial^{\alpha} \omega\right\|^{2}+\varepsilon\left\|\partial^{\alpha} D\right\|^{2}+\varepsilon\left\|\partial^{\alpha} \Omega\right\|^{2}+\frac{a^{2}}{\varepsilon}\left\|\partial^{\alpha} \rho\right\|^{2}\right) \\
& E_{m}^{\varepsilon}(t)=\sum_{|\alpha| \leq m} E_{\alpha, m}^{\varepsilon} .
\end{aligned}
$$

Note that since $\Sigma^{\varepsilon} \mathcal{Q}(\mathcal{U}, \xi)$ and $\Sigma^{\varepsilon} \mathcal{A}(t, x, \xi)$ are symmetric, we can consider (6) as a quasilinear hyperbolic system with zero order terms (a linear one coming from $\mathcal{L}$ and a semilinear one coming from $\mathcal{S}$ which are pseudo-differential operators of order zero when acting on $\mathcal{U}$. Consequently, for $\varepsilon>0$ fixed, we have a result of local existence and uniqueness of strong solutions in $C_{T}\left(H^{m}\right)$; see for instance [1, 17]. This allows us to define $T^{\varepsilon}$ as the largest time such that

$$
E_{m}^{\varepsilon}(t) \leq M_{\varepsilon}, \forall t \in\left[0, T^{\varepsilon}\right),
$$

where $M_{\varepsilon}$ which is such that $M_{\varepsilon} \rightarrow 0$ when $\varepsilon$ goes to zero will be chosen carefully later. To achieve the proof of Theorem 1, and in particular inequality (4), it is sufficient to establish that $T^{\varepsilon} \geq T$, which will be proved by showing that in (9) the equality cannot be reached for $T^{\varepsilon}<T$ thanks to a good choice of $M_{\varepsilon}$.

Before performing the energy estimate, we apply the operator $\partial^{\alpha}$ for $|\alpha| \leq m$ to (6), to obtain

$$
\begin{aligned}
\mathcal{A}_{0}^{\varepsilon} \partial_{t} \partial^{\alpha} \mathcal{U}+\mathcal{A}\left(t, x, \partial_{x}\right) \partial^{\alpha} \mathcal{U} & +\frac{1}{\varepsilon} \mathcal{K}^{\varepsilon} \partial^{\alpha} \mathcal{U}+\mathcal{D} \partial^{\alpha} \mathcal{U}+\partial^{\alpha} \mathcal{N}(\mathcal{U}) \\
& =\partial^{\alpha} \mathcal{L}\left(t, x, \partial_{x}\right) \mathcal{U}+\varepsilon R+\left[\partial^{\alpha}, \mathcal{A}\left(t, x, \partial_{x}\right)\right] \mathcal{U}
\end{aligned}
$$

Now, we proceed to perform the energy estimates for (10) in a classical way by taking the scalar product of system (10) with $\Sigma^{\varepsilon} \partial^{\alpha} \mathcal{U}$. Along the proof, we shall denote by $C$ a number independent of $\varepsilon$, which actually may change from line to line, and by $\mathcal{C}(\cdot)$ a nondecreasing function. Moreover $(\cdot, \cdot)$ and $\|\cdot\|$ stand for the usual $L^{2}$ scalar product and norm, $\|\cdot\|_{m}$ is the usual $H^{m}$ Sobolev norm and $\|\cdot\|_{m, \infty}$ is the usual $W^{m, \infty}$ norm.

Let us start the estimate of each term. First, since $\Sigma^{\varepsilon} \mathcal{A}(x, \xi)$ is symmetric and $\operatorname{div} u^{N S}=0$, we have that

$$
\left|\left(\Sigma^{\varepsilon} \mathcal{A}\left(x, \partial_{x}\right) \partial^{\alpha} \mathcal{U}, \partial^{\alpha} \mathcal{U}\right)\right|=\left.\left|\frac{a^{2}}{\varepsilon} \int \operatorname{div}\left(u^{N S}\right)\right| \partial^{\alpha} \rho\right|^{2} d x \mid=0 .
$$

Next, since $\Sigma^{\varepsilon} \mathcal{K}^{\varepsilon}$ is skew-symmetric, we have that

$$
\left(\Sigma^{\varepsilon} \mathcal{K}^{\varepsilon} \partial^{\alpha} \mathcal{U}, \partial^{\alpha} \mathcal{U}\right)=0
$$

Also, we have

$$
\left(\Sigma^{\varepsilon} \mathcal{D} \partial^{\alpha} \mathcal{U}, \partial^{\alpha} \mathcal{U}\right)=\left\|\partial^{\alpha} D\right\|^{2}+\left\|\partial^{\alpha} \Omega\right\|^{2} .
$$

Now we observe that, since $\int_{\mathbb{T}^{d}} u=0$, we have the inequality

$$
\|u\|_{m+1}^{2} \leq C\left(\|d\|_{m}^{2}+\|\omega\|_{m}^{2}\right) \leq C\|\nabla u\|_{m}^{2}
$$


Hence, the Young inequality gives

$$
\begin{aligned}
\left(\Sigma^{\varepsilon} \partial^{\alpha} \mathcal{L}, \partial^{\alpha} \mathcal{U}\right) & =\int \partial^{\alpha} \operatorname{div}\left(u^{N S} \otimes u+u \otimes u^{N S}\right) \partial^{\alpha} D \\
& +\int \partial^{\alpha} \operatorname{curl}\left(u^{N S} \otimes u+u \otimes u^{N S}\right) \partial^{\alpha} \Omega \\
& \leq \frac{1}{4}\left(\left\|\partial^{\alpha} D\right\|^{2}+\left\|\partial^{\alpha} \Omega\right\|^{2}\right)+\mathcal{C}\left(\left\|u^{N S}\right\|_{m+1, \infty}\right)\left(\|\omega\|_{m}^{2}+\|d\|_{m}^{2}\right) \\
& \leq \frac{1}{4}\left(\left\|\partial^{\alpha} D\right\|^{2}+\left\|\partial^{\alpha} \Omega\right\|^{2}\right)+\mathcal{C}\left(\left\|u^{N S}\right\|_{m+1, \infty}\right) E_{m}^{\varepsilon} .
\end{aligned}
$$

To give the estimate of the term $\left(\Sigma^{\varepsilon} \partial^{\alpha} \mathcal{N}, \partial^{\alpha} \mathcal{U}\right)$, we split it in several terms. The first one is given by, for $m>d / 2$,

$$
\begin{aligned}
\int\left(\partial^{\alpha} \operatorname{div}(u \otimes u)\right. & \left.\partial^{\alpha} D+\partial^{\alpha} \operatorname{curl}(u \otimes u) \partial^{\alpha} \Omega\right) \\
& \leq \frac{1}{4}\left(\left\|\partial^{\alpha} D\right\|^{2}+\left\|\partial^{\alpha} \Omega\right\|^{2}\right)+C\|u \otimes u\|_{m+1}^{2} \\
& \leq \frac{1}{4}\left(\left\|\partial^{\alpha} D\right\|^{2}+\left\|\partial^{\alpha} \Omega\right\|^{2}\right)+\left(E_{m}^{\varepsilon}\right)^{2} .
\end{aligned}
$$

The second one is

$$
\begin{aligned}
& \frac{1}{\varepsilon} \int \partial^{\alpha} \operatorname{div}(\rho u) \partial^{\alpha} \rho \\
& =\frac{1}{\varepsilon} \int u \cdot \nabla \partial^{\alpha} \rho \partial^{\alpha} \rho+\left[\partial^{\alpha}, u \cdot \nabla\right] \rho \partial^{\alpha} \rho+\partial^{\alpha}(\rho \operatorname{div} u) \partial^{\alpha} \rho \\
& =I+I I+I I I .
\end{aligned}
$$

By an integration by parts, we have $I=-\frac{1}{\varepsilon} \int \operatorname{div} u\left|\partial^{\alpha} \rho\right|^{2}$, hence

$$
I \leq\|\operatorname{div} u\|_{0, \infty} E_{m}^{\varepsilon} \leq\left(E_{m}^{\varepsilon}\right)^{\frac{3}{2}} .
$$

The terms $I I$ and $I I I$ are easily estimated by

$$
\begin{aligned}
I I & \leq C\|u\|_{m} \frac{\|\rho\|_{m}^{2}}{\varepsilon} \leq C\left(E_{m}^{\varepsilon}\right)^{\frac{3}{2}}, \\
I I I & \leq C\left(\|u\|_{m}+\|\nabla u\|_{m}\right) \frac{\|\rho\|_{m}^{2}}{\varepsilon} \leq C\left(\|d\|_{m}+\|\omega\|_{m}\right) \frac{\|\rho\|_{m}^{2}}{\varepsilon} \leq C\left(E_{m}^{\varepsilon}\right)^{\frac{3}{2}} .
\end{aligned}
$$

Therefore we obtain the estimate

$$
\frac{1}{\varepsilon} \int \partial^{\alpha} \operatorname{div}(\rho u) \partial^{\alpha} \rho \leq C\left(E_{m}^{\varepsilon}\right)^{\frac{3}{2}}
$$

To estimate the commutator, we have

$$
\begin{aligned}
& \left(\Sigma^{\varepsilon}\left[\partial^{\alpha}, \mathcal{A}\left(x, \partial_{x}\right)\right] \mathcal{U}, \mathcal{U}\right)=\frac{1}{\varepsilon} \int\left[\partial^{\alpha}, u^{N S} \cdot \nabla\right] \rho \partial^{\alpha} \rho d x \\
& \quad \leq C \frac{\|\rho\|_{m}}{\varepsilon}\left(\left\|u^{N S}\right\|_{m}\|\nabla \rho\|_{0, \infty}+\left\|\nabla u^{N S}\right\|_{0, \infty}\|\nabla \rho\|_{m-1}\right) \\
& \quad \leq \mathcal{C}\left(\left\|u^{N S}\right\|_{1, \infty}+\left\|u^{N S}\right\|_{m}\right) E_{m}^{\varepsilon} .
\end{aligned}
$$

To get the last line, we have used the fact that $m>1+d / 2$. 
Finally, we have that (18)

$$
\begin{aligned}
\varepsilon\left|\left(\Sigma^{\varepsilon} \partial^{\alpha} R, \partial^{\alpha} \mathcal{U}\right)\right| & \leq \varepsilon\left\|\nabla u^{N S} \cdot \nabla u^{N S}\right\|_{m}\|d\|_{m}+\left\|\partial_{t} \operatorname{div} V^{N S}\right\|_{m}\|D\|_{m} \\
& +\left\|\partial_{t} \operatorname{curl} V^{N S}\right\|_{m}\|\Omega\|_{m} \\
& \leq\|d\|_{m}^{2}+\frac{1}{4}\left(\|D\|_{m}^{2}+\|\Omega\|_{m}^{2}\right)+\varepsilon^{2} \mathcal{C}\left(\left\|u^{N S}\right\|_{m+1}+\left\|\partial_{t} V^{N S}\right\|_{m}\right) \\
& \leq E_{m}^{\varepsilon}+\frac{1}{4}\left(\|D\|_{m}^{2}+\left\|\Omega_{m}\right\|^{2}\right)+\varepsilon^{2} \mathcal{C}\left(\left\|u^{N S}\right\|_{m+3}\right) .
\end{aligned}
$$

Note that, to get the last line, we have used the explicit expression (2) of $V^{N S}$ and the Navier-Stokes equations to replace the time derivative by spatial ones.

Now, we collect all the previous estimates (11)-(18), and we sum over $\alpha$ to find

$$
\begin{aligned}
\frac{d}{d t} E_{m}^{\varepsilon}+\frac{1}{4}\left(\|D\|_{m}^{2}+\|\Omega\|_{m}^{2}\right) & \leq \varepsilon^{2} \mathcal{C}\left(\left\|u^{N S}\right\|_{m+3}\right)+\mathcal{C}\left(\left\|u^{N S}\right\|_{m+3}\right) E_{m}^{\varepsilon} \\
& +\left(E_{m}^{\varepsilon}\right)^{2}+C\left(E_{m}^{\varepsilon}\right)^{\frac{3}{2}} \\
& \leq \varepsilon^{2} \mathcal{C}\left(\left\|u^{N S}\right\|_{m+3}\right)+\mathcal{C}\left(\left\|u^{N S}\right\|_{m+3}\right) E_{m}^{\varepsilon}+\left(E_{m}^{\varepsilon}\right)^{2}
\end{aligned}
$$

By using (9), we get with $M_{\varepsilon} \leq 1$ that

$$
\frac{d}{d t} E_{m}^{\varepsilon} \leq \mathcal{C}_{m+3} \varepsilon^{2}+\mathcal{C}_{m+3} E_{m}^{\varepsilon}, \forall t \in\left[0, T^{\varepsilon}\right)
$$

where $\mathcal{C}_{m+3}=\mathcal{C}\left(\left\|u^{N S}\right\|_{m+3}\right)$, and hence by the Gronwall inequality we get that

$$
E_{m}^{\varepsilon}(t) \leq\left(\alpha_{m}(\varepsilon)+t \mathcal{C}_{m+3} \varepsilon^{2}\right) e^{\mathcal{C}_{m+3} t}, \forall t \in\left[0, T^{\varepsilon}\right) .
$$

Consequently, if we choose $M_{\varepsilon}=\left(\alpha_{m}(\varepsilon)+T \mathcal{C}_{m+3} \varepsilon^{2}\right)^{\frac{1}{2}}$, we see that we cannot reach equality in (9) for $T^{\varepsilon}<T$. This proves that $T^{\varepsilon} \geq T$ and that (19) is valid on [0,T].

\section{ACKNOWLEDGEMENT}

Support by the European network HYKE funded by the EC as contract HPRNCT-2002-00282 is acknowledged.

\section{REFERENCES}

[1] S. Alinhac and P. Gérard. Opérateurs pseudo-différentiels et théorème de Nash-Moser. Savoirs Actuels. [Current Scholarship]. InterEditions, Paris, 1991. MR.1172111 (93g:35001)

[2] D. Aregba-Driollet, R. Natalini, and S.Q. Tang. Diffusive kinetic explicit schemes for nonlinear degenerate parabolic systems. Math. Comp. 73 (2004) 63-94. MR2034111 (2004m:65136)

[3] M.K. Banda, A. Klar, L. Pareschi, and M. Seaid. Compressible and Incompressible Limits for Hyperbolic Systems with Relaxation Journal of Computational and Applied Mathematics 168 (2004) 41-52. MR2078995 (2005g:35239)

[4] F. Bouchut, F. Golse, and M. Pulvirenti. Kinetic equations and asymptotic theory. Series in Appl. Math., Gauthiers-Villars, 2000. MR2065070 (2005d:82102)

[5] F. Bouchut, F. Guarguaglini, and R. Natalini. Discrete kinetic approximation to multidimensional parabolic equations. Indiana Univ. Math. J., 49:723-749, 2000. MR.1793689 (2001k:35162)

[6] Y. Brenier, Convergence of the Vlasov-Poisson system to the incompressible Euler equations. Comm. Partial Differential Equations 25 (2000), no. 3-4, 737-754. MR:1748352|(2001c:76124)

[7] Y. Brenier, R. Natalini, and M. Puel. On a relaxation approximation of the incompressible Navier-Stokes equations. Proc. Amer. Math. Soc., 132(4):1021-1028 (electronic), 2004. MR2045417 (2005b:35218) 
[8] S. Cordier and E. Grenier. Quasineutral limit of an Euler-Poisson system arising from plasma physics. Comm. Partial Differential Equations, 25(5-6):1099-1113, 2000. MR1759803 (2001c:82078)

[9] D. Donatelli and P. Marcati. Convergence of singular limits for multi-D semilinear hyperbolic systems to parabolic systems. Trans. Amer. Math. Soc., 356(5):2093-2121 (electronic), 2004. MR2031055 (2004k:35240)

[10] D. Donatelli and P. Marcati. Diffusive singular limits and 3-D incompressible Navier-Stokes equation. To appear in Proceedings of HYP 2004 Tenth International Conference on Hyperbolic Problems Theory, Numerics, Applications, Osaka, Japan, Yokoama Publishers, Inc., 2005.

[11] E. Grenier. Defect measures of the Vlasov-Poisson system in the quasineutral regime. Commun. Partial Differ. Equations 20, No.7-8, 1189-1215, 1995.

[12] E. Grenier. Oscillations in quasineutral plasmas. Commun. Partial Differ. Equations 21, No.3-4, 363-394, 1996. MR1335748 (96k:35146)

[13] Th. Katsaounis, Ch. Makridakis, and C. Simeoni. Relaxation methods and finite element schemes for the incompressible Navier-Stokes equations. Preprint 2004.

[14] G. Loeper. Quasineutral limit for the Euler-Poisson and Euler-Monge-Ampère systems. Preprint, 2003; to appear in Comm. Partial Differential Equations.

[15] P. Marcati, A. Milani, and P. Secchi. Singular convergence of weak solutions for a quasilinear nonhomogeneous hyperbolic system. Manuscripta Math., 60:49-69, 1988. MR0920759 (89f:35127)

[16] P. Marcati and B. Rubino. Hyperbolic to parabolic relaxation theory for quasilinear first order systems. J. Differential Equations, 162(2):359-399, 2000. MR.1751710(2001d:35125)

[17] M. E. Taylor. Partial differential equations. III, volume 117 of Applied Mathematical Sciences. Springer-Verlag, New York, 1997. Nonlinear equations, Corrected reprint of the 1996 original. MR1477408 (98k:35001)

[18] Shu Wang. Quasineutral limit of Euler-Poisson system with and without viscosity. Comm. Partial Differential Equations, 29(3-4):419-456, 2004. MR2041602 (2005i:35225)

Istituto per le Applicazioni del Calcolo "Mauro Picone", Consiglio Nazionale delle Ricerche, Viale del Policlinico, 137, I-00161 Roma, Italy

E-mail address: r.natalini@iac.cnr.it

CNRS, Laboratoire J.-A Dieudonne, UMr 6621, Universite de Nice, Parc Valrose, F-06108 Nice Cedex 02, France

E-mail address: frousset@math.unice.fr 